\title{
1 Understanding Climate-Fire-Ecosystem Interactions Using 2 CESM-RESFire and Implications for Decadal Climate 3 Variability
}

4 Yufei Zou ${ }^{1 \dagger}$, Yuhang Wang ${ }^{1}$, Yun Qian², Hanqin Tian ${ }^{3}$, Jia Yang ${ }^{4}$, Ernesto Alvarado

$5 \quad{ }^{1}$ School of Earth and Atmospheric Sciences, Georgia Institute of Technology, Atlanta, GA 30332, USA.

$6 \quad{ }^{2}$ Pacific Northwest National Laboratory, Richland, WA 99354, USA.

7 International Centre for Climate and Global Change Research, School of Forestry and Wildlife Sciences, Auburn 8 University, AL 36849, USA.

$9{ }^{4}$ College of Forest Resources/Forest and Wildlife Research Center, Mississippi State University, MS 39762, USA.

$10{ }^{5}$ School of Environmental and Forest Sciences, University of Washington, Seattle, WA 98195, USA.

$11 \dagger$ Now at the School of Environmental and Forest Sciences, University of Washington, Seattle, WA 98195, USA.

12 Correspondence to: Yuhang Wang (yuhang.wang@eas.gatech.edu) and Yufei Zou (yzou2017@uw.edu)

\section{Supplement}

14 The AERONET network did not provide AOT measurements at $550 \mathrm{~nm}$ wavelength. For direct comparison with the

15 model results, we estimated AERONET AOT at $550 \mathrm{~nm}$ by interpolating the measurements at two closest

16 wavelengths at $500 \mathrm{~nm}$ and $675 \mathrm{~nm}$. Specifically, the optical thickness of aerosols and the wavelength of light

17 satisfies the power law (Ångström, 1929) in Eq. (S1):

$18 \frac{\tau_{\lambda}}{\tau_{\lambda_{0}}}=\left(\frac{\lambda}{\lambda_{0}}\right)^{-\alpha},(\mathrm{S} 1)$

19 where $\tau_{\lambda}$ is the optical thickness at wavelength $\lambda, \tau_{\lambda_{0}}$ is the optical thickness at the reference wavelength $\lambda_{0}$, and $\alpha$

20 is the Ångström exponent.

21 We first calculated the Ångström exponent based on the optical thickness measured at $500 \mathrm{~nm}$ and $675 \mathrm{~nm}$, then

22 estimated the optical thickness at $550 \mathrm{~nm}$ using Eq. (S1) and AOT at $500 \mathrm{~nm}$ as the reference. The estimation

23 equation is shown in Eq. (S2):

24

$\tau_{550}=\tau_{500}\left(\frac{550}{500}\right)^{-\alpha}$, where $\alpha=-\frac{\log \frac{\tau_{675}}{\tau_{500}}}{\log \frac{675}{500}}$

25

26

Reference

27 Ångström, A.: On the Atmospheric Transmission of Sun Radiation and on Dust in the Air, Geografiska Annaler, 11, 